\title{
Some Unified Integrals Pertaining to Srivastava Polynomials
}

Vijay Kumar Singhal, Nawal Kishor Jangid, Amber Srivastava

Department of Mathematics, Swami Keshvanand Institute of Technology, Management \& Gramothan, Jaipur-302017 (INDIA)

Email: vijay.singhal@skit.ac.in,nawal.jangid@skit.ac.in,amber@skit.ac.in Received 10.09.2021, received in revised form 2.10.2021, accepted 4.10.2021 doi: 10.47904/IJSKIT.11.2.2021.81-84

\begin{abstract}
The present work involves study of three unified integrals containing product of Srivastava polynomials, Mittag-Leffler function, Fox's H-function and generalized Wright function. Being unified in nature, the main integrals established here yield a number of known and new results as special cases. For the sake of illustration, some particular cases are recorded here.
\end{abstract}

Keywords- Fox's H-function, Mittag-Leffler function, Srivastava Polynomials.

\section{INTRODUCTION}

We recall here the following representations for ready reference during the study:

The generalized Wright function [8] occurring in the paper is expressed in series form as

$$
\begin{gathered}
{ }_{p} \Psi_{q}(z) \equiv{ }_{p} \Psi_{q}\left[z \mid \begin{array}{l}
\left(a_{i}, \alpha_{i}\right)_{1, p} \\
\left(b_{j}, \beta_{j}\right)_{1, q}
\end{array}\right] \\
=\sum_{k=0}^{\infty} \frac{\prod_{i=1}^{p} \Gamma\left(a_{i}+\alpha_{i} k\right)}{\prod_{j=1}^{q} \Gamma\left(b_{j}+\beta_{j} k\right)} \frac{z^{k}}{k !} \\
z \in \mathbb{C}, a_{i}, b_{j} \in \mathbb{C} \text { and } \alpha_{i}, \beta_{j} ; \alpha_{i}, \beta_{j} \in \mathbb{R}, \\
i=1,2, \ldots, p ; j=1,2, \ldots q ; \sum_{j=1}^{q} \beta_{j}-\sum_{i=1}^{p} \alpha_{i}>-1
\end{gathered}
$$

The following expression for the Mittag-Leffler function [2, ChapterIII], [3, Vol.3, Section 18.1] is used here

$$
\mathrm{E}_{\alpha^{\prime}, \beta^{\prime}}(z)=\sum_{k=0}^{\infty} \frac{z^{k}}{\Gamma\left(\alpha^{\prime} k+\beta^{\prime}\right)} \quad ;\left(z, \beta^{\prime} \in \square, \alpha^{\prime}>0\right)
$$

Further, the following representation of Srivastava polynomials [12] is also used during the present study

$$
\begin{aligned}
& S_{N^{\prime}}^{M^{\prime}}[x]=\sum_{k=0}^{\left[N^{\prime} / M^{\prime}\right]}\left(-N^{\prime}\right)_{M^{\prime} k} A_{N, k} \frac{x^{k}}{k !}, \\
& N^{\prime}=0,1,2 \ldots
\end{aligned}
$$

Where $A_{N^{\prime}, k}\left(N^{\prime}, k \geq 0\right)$ are arbitrary constants, real or complex and $M^{\prime}$ being arbitrary positive integer The Fox's $H$-function appearing in this paper is defined as

$$
\begin{aligned}
& H_{B, D}^{A, C}[z]=H_{B, D}^{A, C}\left[z \mid \begin{array}{l}
\left(a_{i} ; \alpha_{i}\right)_{1, B} \\
\left(b_{j}, \beta_{j}\right)_{1, D}
\end{array}\right] \\
& =\frac{1}{2 \pi i} \int_{L} \Phi(\xi) z^{-\xi} d \xi(1.6) \\
& \text { where } \\
& \qquad(\xi)=\frac{\prod_{j=1}^{A} \Gamma\left(b_{j}+\beta_{j} \xi\right) \prod_{j=1}^{C} \Gamma\left(1-a_{j}-\alpha_{j} \xi\right)}{\prod_{j=A+1}^{D} \Gamma\left(1-b_{j}-\beta_{j} \xi\right) \prod_{j=C+1}^{B} \Gamma\left(a_{j}+\alpha_{j} \xi\right)}
\end{aligned}
$$

For all details of this function one can see the works [9, 10, 12, 13, and 14].

The above functions find their applicationsin the areas like analytical solar and stellar models, nuclear reaction rate theory, gravitational instability problems, pathway analysis etc. Unified integrals involving special functions play a pivotal rolein various fields of applied sciences. This has been motivating factor for the researchers to study such integrals $[4,5,6]$. The present paper deals with the study of three unified integrals pertaining to $\mathrm{H}$ function and Srivastava polynomials. On account of the general and unified nature of the functions involved, these integrals yield a number of new and known results which may prove to be useful in dealing with the problems of science and engineering as well.

\section{MAIN RESULTS}

\section{First Integral}

$$
\begin{array}{r}
\int_{0}^{\infty}\left[\left(a x+\frac{b}{x}\right)^{2}+c\right]^{\eta-1} S_{N}^{M}\left[y\left\{\left(a x+\frac{b}{x}\right)^{2}+c\right\}^{\sigma}\right] \\
\times \mathrm{E}_{\alpha^{\prime}, \beta^{\prime}}\left[z\left\{\left(a x+\frac{b}{x}\right)^{2}+c\right\}^{\rho}\right] \\
\times H_{B, D}^{A, C}\left[z\left\{\left(a x+\frac{b}{x}\right)^{2}+c\right\}^{v} \mid\left(\begin{array}{l}
\left(a_{i} ; \alpha_{i}\right)_{1, B} \\
\left(b_{j}, \beta_{j}\right)_{1, D}
\end{array}\right] d x\right.
\end{array}
$$




$$
\begin{aligned}
& =\frac{\sqrt{\pi}}{2 a(4 a b+c)^{-\eta+1 / 2}} \sum_{k=0}^{[N / M]} \sum_{k^{\prime}=0}^{\infty}(-N) M k \\
& \times A_{N, k} \frac{y^{k}}{k !(4 a b+c)^{-\sigma k}} \frac{z^{k^{\prime}}}{\Gamma\left(\alpha^{\prime} k^{\prime}+\beta^{\prime}\right)(4 a b+c)^{-\rho k^{\prime}}} \\
& \times H_{B+1, D\left[z(4 a b+c)^{-v}\right.}^{A+1, C[}\left[\begin{array}{l}
\left(a_{i} ; \alpha_{i}\right)_{1, B},\left(1-\eta-\sigma k-\rho k^{\prime} ; v\right) \\
\left(1 / 2-\eta-\sigma k-\rho k^{\prime} ; v\right),\left(b_{i} ; \beta_{i}\right) 1, D
\end{array}\right]
\end{aligned}
$$

Where

$$
\begin{aligned}
& \alpha>0, \beta \in \square, z \in \square ; \eta, \sigma, \rho>0 \\
& \operatorname{Re}\left(\eta+\sigma+\rho+v \frac{b_{j}}{\beta_{j}}\right)>0 ; j=1,2, \ldots, A ; T>0 \\
& |\arg (z)|<\frac{1}{2} T \pi, \\
& \text { where } \\
& \quad T_{i}=\sum_{j=1}^{C} a_{j}-\sum_{j=C+1}^{B} a_{j}+\sum_{j=1}^{A} b_{j}-\sum_{j=A+1}^{D} b_{j}
\end{aligned}
$$

Proof:

Firstly, we use the expressions (1.2), (1.3) and (1.4) in the LHS. Then on interchanging the order of summation and integration we readily arrive at the desired result by using the following known integral [7, pp.328]

$$
\begin{aligned}
& \int_{0}^{\infty}\left[\left(a x+\frac{b}{x}\right)^{2}+c\right]^{-p-1} d x=\frac{1}{2} \frac{\mathrm{B}\left(\mathrm{p}+\frac{1}{2}, \frac{1}{2}\right)}{a(4 a b+c)^{\mathrm{p}+\frac{1}{2}}} \\
& a>0, b>0, c>-4 a b, p>-\frac{1}{2}
\end{aligned}
$$

\section{Second integral}

$$
\begin{array}{r}
\int_{0}^{\infty}\left[\left(a x+\frac{b}{x}\right)^{2}+c\right]^{\eta-1} S_{N}^{M}\left[y\left\{\left(a x+\frac{b}{x}\right)^{2}+c\right\}^{\sigma}\right] \\
\times{ }_{p} \Psi_{q}\left[z\left\{\left(a x+\frac{b}{x}\right)^{2}+c\right\}^{\lambda}\right] \\
\times H_{B, D}^{A, C}\left[z\left\{\left(a x+\frac{b}{x}\right)^{2}+c\right\}^{v} \mid\left(\begin{array}{l}
\left(a_{i} ; \alpha_{i}\right)_{1, B} \\
\left(b_{j}, \beta_{j}\right)_{1, D}
\end{array}\right] d x\right.
\end{array}
$$

$$
\begin{gathered}
=\frac{\sqrt{\pi}}{2 a(4 a b+c)^{-\eta+1 / 2}} \sum_{k=0}^{[N / M]} \sum_{K^{\prime}=0}^{\infty}(-N)_{M k} A_{N, k} \\
\times \frac{y^{k}}{k !(4 a b+c)^{-\sigma k}} \\
\times \frac{\prod_{i=1}^{p} \Gamma\left(a_{i}{ }^{\prime}+\alpha_{i}^{\prime} k^{\prime}\right) z^{k^{\prime}}}{\prod_{j=1}^{q} \Gamma\left(b_{j}{ }^{\prime}+\beta_{j}{ }^{\prime} k^{\prime}\right) k^{\prime} !(4 a b+c)^{-\lambda k^{\prime}}} \\
\times H_{B+1, D}^{A+1, C}\left[z(4 a b+c)^{-v}\right. \\
\left.\quad \begin{array}{c}
\left(a_{i}, \alpha_{i}\right)_{1, B},\left(1-\eta-\sigma k-\lambda k^{\prime} ; v\right) \\
\left(1 / 2-\eta-\sigma k-\lambda k^{\prime} ; v\right),\left(b_{j} ; \beta_{j}\right)_{1, D}
\end{array}\right]
\end{gathered}
$$

Where,

$$
\begin{aligned}
& a_{i}, b_{j} \in \square ; z \in \square ; \alpha_{i}, \beta_{j} \in \square,\left(\alpha_{i}, \beta_{j} \neq 0\right) ; \\
& i=1,2, \ldots, p ; j=1,2, \ldots, q \\
& \operatorname{Re}\left(\eta+\sigma+\lambda+v \frac{b_{j}}{\beta_{j}}\right)>0 ; j=1,2, \ldots, A ;
\end{aligned}
$$

$T>0 ; \quad|\arg (z)|<\frac{1}{2} T \pi$,

where

$$
T_{i}=\sum_{j=1}^{C} a_{j}-\sum_{j=C+1}^{B} a_{j}+\sum_{j=1}^{A} b_{j}-\sum_{j=A+1}^{D} b_{j}
$$

\section{Proof:}

Firstly, we use the expressions (1.1), (1.3) and (1.4) in the LHS. Then on interchanging the order of summation and integration we get the desired result in view of the known integral [7, pp.328]

\section{Third Integral}

$$
\int_{-1}^{1}(1-x)^{\rho}(1+x)^{\sigma} S_{N}^{M}\left[y(1-x)^{n}(1+x)^{m}\right]
$$

$$
\begin{gathered}
\times{ }_{p} \Psi_{q}\left[z(1-x)^{\mu}(1+x)^{\lambda}\right] \\
\times H_{B, D}^{A, C}\left[z(1-x)^{\nu}(1+x)^{\delta} \mid \begin{array}{l}
\left(a_{i} ; \alpha_{i}\right)_{1, B} \\
\left(b_{j}, \beta_{j}\right)_{1, D}
\end{array}\right] d x
\end{gathered}
$$




$$
\begin{aligned}
& =2^{\rho+\sigma+1} \sum_{k=0}^{[N / M]}(-N)_{M k} A_{N, k} \frac{y^{k}}{k !} 2^{(m+n) k} \\
& \times \sum_{k^{\prime}=0}^{\infty} \frac{\prod_{i=1}^{p} \Gamma\left(a_{i}{ }^{\prime}+\alpha_{i}{ }^{\prime} k^{\prime}\right)}{\prod_{j=1}^{q} \Gamma\left(b_{j}{ }^{\prime}+\beta_{j}{ }^{\prime} k^{\prime}\right)} \times \frac{2^{(\mu+\lambda) k^{\prime}} z^{k^{\prime}}}{k^{\prime \prime} !} \\
& \times H_{B+2, D+1}^{A, C+2}\left[z .2^{v+\delta} \mid \begin{array}{c}
\left(-\rho-n k-\mu k^{\prime} ; v\right), \\
\left(b_{j}, \beta_{j}\right)_{1, D},
\end{array}\right. \\
& \left(-\sigma-m k-\lambda k^{\prime} ; \delta\right),\left(a_{i}, \alpha_{i}\right)_{1, B} \\
& \left(-1-\sigma-\rho-(m+n) k-(\mu+\lambda) k^{\prime}, v+\delta\right]
\end{aligned}
$$

Where $m, n>0 ; \alpha_{i}, \beta_{j} \in \square,\left(\alpha_{i}, \beta_{j} \neq 0\right)$;

Where, $i=1,2, \ldots, p ; j=1,2, \ldots, q$

$\operatorname{Re}\left(\rho+n+\mu+v \frac{b_{j}}{\beta_{j}}\right)>0 ; j=1,2, \ldots, A$;

$\operatorname{Re}\left(\sigma+m+\lambda+\delta \frac{b_{j}}{\beta_{j}}\right)>0 ; j=1,2, \ldots, A$

$T>0,|\arg (z)|<\frac{1}{2} T \pi$,

where

$$
T_{i}=\sum_{j=1}^{C} a_{j}-\sum_{j=C+1}^{B} a_{j}+\sum_{j=1}^{A} b_{j}-\sum_{j=A+1}^{D} b_{j}
$$

Proof:

Following the steps similar to those used in establishing the second integral and then using the known result [7 pp.316], we arrive at the desired result.

\section{Special cases:}

(i) Taking

$$
M=1, A_{N, k}=\left(\begin{array}{c}
N+\alpha^{\prime} \\
N
\end{array}\right) \frac{\left(\alpha^{\prime}+\beta^{\prime}+N+1\right)_{k}}{\left(\alpha^{\prime}+1\right)_{k}},
$$

result (2.1) takes the form

$$
\begin{aligned}
& \int_{0}^{\infty}\left[\left(a x+\frac{b}{x}\right)^{2}+c\right]^{\eta-1} \\
& \times P_{N}^{\left(\alpha^{\prime \prime}, \beta^{\prime \prime}\right)}\left[1-2 y\left\{\left(a x+\frac{b}{x}\right)^{2}+c\right\}^{\sigma}\right] \\
& \times E_{\alpha^{\prime}, \beta^{\prime}}\left[z\left\{\left(a x+\frac{b}{x}\right)^{2}+c\right\}^{\rho}\right] \\
& \times H_{B, D}^{A, C}\left[z\left\{\left(a x+\frac{b}{x}\right)^{2}+c\right\}^{v} \mid \begin{array}{l}
\left(a_{i} ; \alpha_{i}\right)_{1, B} \\
\left(b_{j}, \beta_{j}\right)_{1, D}
\end{array}\right] d x \\
& =\frac{\sqrt{\pi}}{2 a(4 a b+c)^{-\eta+1 / 2}} \sum_{k=0}^{N}(-N)_{k}\left(\begin{array}{c}
N+\alpha^{\prime} \\
N
\end{array}\right) \\
& \times \frac{\left(\alpha^{\prime \prime}+\beta^{\prime \prime}+N+1\right)_{k}}{\left(\alpha^{\prime \prime}+1\right)_{k}} \frac{y^{k}}{k !(4 a b+c)^{-\sigma k}} \\
& \times H_{B+1, D}^{A+1, C}\left[z(4 a b+c)^{-v}\right. \\
& \left.\mid \begin{array}{c}
\left(a_{i} ; \alpha_{i}\right)_{1, B},\left(1-\eta-\sigma k-\rho k^{\prime} ; v\right) \\
\left(1 / 2-\eta-\sigma k-\rho k^{\prime} ; v\right),\left(b_{i} ; \beta_{i}\right)_{1, D}
\end{array}\right]
\end{aligned}
$$

Which exists under the same conditions surrounding the result (2.1)

(ii) If $p=1, q=1, a_{1}=\rho, \alpha_{1}=1, b_{1}=\beta, \beta_{1}=\alpha$ then the result (2.2) takes the form 


$$
\begin{gathered}
\int_{0}^{\infty}\left[\left(a x+\frac{b}{x}\right)^{2}+c\right]^{\eta-1} S_{N}^{M}\left[y\left\{\left(a x+\frac{b}{x}\right)^{2}+c\right\}^{\sigma}\right] \\
\times E_{\alpha, \beta}^{\rho}\left[z\left\{\left(a x+\frac{b}{x}\right)^{2}+c\right\}^{\lambda}\right] d x^{\lambda} \\
=\frac{\sqrt{\pi}}{2 a(4 a b+c)^{-\eta+1 / 2} \Gamma(\rho)} \sum_{k=0}^{[N / M]}(-N)_{M k} A_{N, k} \\
\times \frac{y^{k}}{k !(4 a b+c)^{-\sigma k}} \\
\times H_{B+1, D}^{A+1, C}\left[z(4 a b+c)^{-v}\right. \\
\left.\mid \begin{array}{c}
\left(a_{i}, \alpha_{i}\right)_{1, B},\left(1-\eta-\sigma k-\lambda k^{\prime} ; v\right) \\
\left(1 / 2-\eta-\sigma k-\lambda k^{\prime} ; v\right),\left(b_{j} ; \beta_{j}\right)_{1, D}
\end{array}\right]
\end{gathered}
$$

Which exists under the same conditions surrounding the result (2.2)

\section{CONCLUSION}

Being unified in nature, the main integrals established here, yield several new findings.

\section{ACKNOWLEDGEMENT}

Authors are thankful to the learned refrees for the valuable suggestions towards the improvement of the present paper.

\section{REFERENCES}

Braaksma, B. L. J. (1936). Asymptotic expansions andanalytic continuations for a class of Barnes- integrals. CompositioMathematica, 15, 239-341.

[2] Dzrbashjan, M. M. (1966). Integral transforms and Representations of Functions in the complex Domain (in Russian) Nauka.

[3] Bateman manuscript project, Erdélyi, A., \& Bateman, H.(1953). HigherTranscendental Functions : Based in Part on Notes Left by Harry Bateman and Compiled by the Staff of the Bateman Manuscript Project. McGraw-Hill.

[4] Bhargava, A., Srivastava, A. \& Mukherjee, R (2016). Some finite Integrals involving Srivastava's polynomials and the Aleph function. Kyungpook Mathematical Journal, 56(2), 465-471.

[5] Chaurasia, V.B.L. \& Singhal, Vijay Kumar (2005). Double integration pertaining to certain polynomials. Bull. Cal. Math. Soc, 97,235-244.

[6] Choi, J. \& Agarwal, P. (2013), Certain Unified integrals associated with Bessel functions, Boundary value problems, 95,9

[7] Gradshteyin, I. S., Ryzhik, I. M. (2001), Table of Integrals, series and products, 6/e, Academic Press, New Delhi.

[8] Kilbas, A. A., Saigo, M., \& Trujillo, J. J. (2002). On the generalized Wright function. Fractional Calculus and Applied Analysis, 5(4), 437-460.

[9] Mathai, A. M., \&Haubold, H. J. (2008). Special functions for applied scientists (Vol. 4). New York: Springer.

[10] Mathai, A. M., Saxena, R. K. (1978). The H-function with applications in statistics and other disciplines. John Wiley \& Sons

[11] Prudnikov, A.Brychkov, Yu. Marichev, O. Integrals and Series, Some More Special Functions, vol. 3, Gordon \& Breach, New York (1992).

[12] Srivastava, H. M. (1972). A contour integral involving Fox's H-function. Indian J. Math, 14(1), 1-6.

[13] Srivastava, H. M., Gupta, K. C. \& Goyal, S. P.(1982). The $\mathrm{H}$-functions of one and two variables, with applications. South Asian Publishers.

[14] Srivastava, H. M., \&Kashyap, B. R. K. (1982). Special functions in queuing theory and related stochastic processes. Academic press, 111 fifth ave., New York, NY 10003. 